\title{
Comportement dynamique linéaire et non linéaire des paliers et des joints
}

\author{
Linear and non linear dynamic behaviour of bearings and seals \\ par O. Bonneau \\ Laboratoire de Mécanique des Solides - UMR 6010 CNRS - Université de Poitiers
}

High speed notors set a lot of stability problems especially when the speed of rotation is going through a critical speed. The dynamical behavior of the fluid bearings has, in that case, an important effect. This study presents several models of dynamical behavior of bearing taking into account the flexibility of the shaft. The squeeze film damper (SFD) behavior is also described. This element gives a lot of damping but its behavior is totally non linear.

An approach of active squeeze film damper is presented : a variable clearance SFD or a variable viscosity SFD (fed with electro-rheological fluid) is used to control a flexible shaft.

\section{INTRODUCTION}

La caractérisation du comportement dynamique des lignes d'arbres de turbomachine nécessite la prise en compte des effets des éléments supports. Deux principaux types d'éléments sont couramment utilisés sur ce type de rotors : les paliers fluides et les joints d'étanchéité. Les paliers sont essentiellement chargés de supporter les charges radiales statiques et dynamiques s'exerçant sur l'arbre, les joints d'étanchéité sont chargés de séparer deux zones de fluides présentant un fort gradient de pression et traversées par un arbre tournant. Ces éléments ont bien évidement un rôle qui peut être prépondérant sur la dynamique de l'ensemble (notamment sur les modes de corps solides).

Dans un premier temps nous présenterons les modélisations permettant de caractériser le comportement statique d'un élément fluide, puis dans un deuxième temps nous aborderons les différentes modélisations dynamiques permettant le couplage entre le comportement de la ligne d'arbre et celui des éléments fluides.

La caractérisation d'un élément fluide passe par la résolution dans le film fluide des équations de Navier-Stokes simplifiées. Cette résolution permet d'obtenir la valeur des champs de vitesses et de pression dans le film. Deux cas de figure apparaissent :

— si l'épaisseur du film reste très petite vis-à-vis des autres dimensions, alors cette condition permet de simplifier l'ensemble des équations et d'obtenir dans le cas des fluides newtoniens une équation différentielle elliptique en pression : l'équation de Reynolds. La résolution de cette équation peut s'envisager de différentes manières : par approche analytique (palier court, palier long) ou par résolution numérique (différences finies, éléments finis).

— s'il n'est pas possible de négliger une dimension vis-à-vis des deux autres, nous sommes alors amenés à résoudre le système complet des équations de Navier-Stokes. Cette approche est nécessaire, par exemple, dans le calcul des joints labyrinthes où il existe des rainures de profondeurs importantes séparant deux zones à film mince. Le comportement du fluide dans ces rainures ne peut pas être envisagé de façon simple. En effet il se crée des tourbillons et le champ de pression évolue selon la direction radiale. Cet aspect ne sera pas présenté ici, il fait l'objet du travail de Arghir [1-2].

\section{II — EQUATIONS DE LA LUBRIFICATION}

Le problème général rencontré dans nos applications concerne le calcul du champ de pression dans le fluide de façon à connaître la valeur des actions mécaniques générées par le fluide sur l'arbre. L'épaisseur du film sera considérée comme négligeable vis-à-vis de l'étendue du film lubrifiant.

La figure 1 représente la section droite d'un palier à arc partiel de longueur 1 .

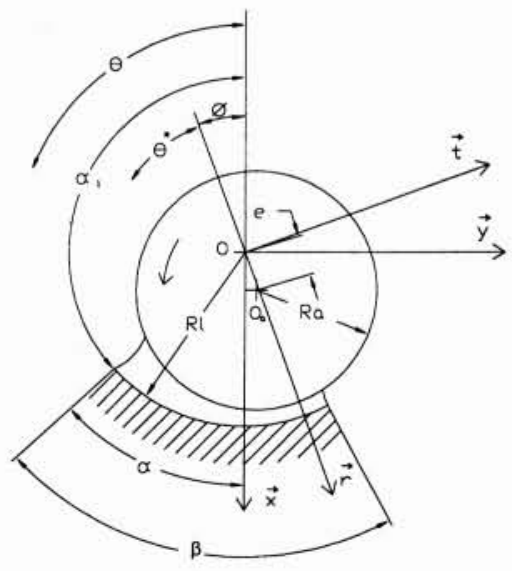

1. Palier à arc partiel. 
$\mathrm{O}_{\mathrm{a}}$ repère le centre de l'arbre et $\mathrm{O}_{\mathrm{c}}$ le centre de courbure de l'arc partiel. Lorsque les axes de l'arbre et du palier sont parallèles (étude sans mésalignement), deux paramètres sont nécessaires pour représenter le centre de l'arbre $\mathrm{O}_{\mathrm{a}}$ vis-à-vis du centre du coussinet $\mathrm{O}_{\mathrm{c}}$.

Nous utilisons généralement deux types de paramétrage pour décrire la position du centre de l'arbre :

- Les coordonnées polaires :

— l'excentricité e $=\left|\mathrm{O}_{3} \mathrm{Oc}\right|$ ou encore l'excentricité relative $\varepsilon=\mathrm{e} / \mathrm{C}$ avec $\mathrm{C}=\mathrm{R}_{\mathrm{c}}-\mathrm{R}_{\mathrm{a}}\left(\mathrm{C}\right.$ représente le jeu radial, $\mathrm{R}_{\mathrm{c}}$ le rayon de courbure du coussinet et $\mathrm{R}_{\mathrm{a}}$ celui de l'arbre).

et

- l'angle de calage $\phi$ qui représente l'angle que fait la ligne des centres $\mathrm{O}_{\mathrm{c}} \mathrm{O}_{\mathrm{a}}$ avec une direction fixe arbitraire $\vec{x}$. Le début du lobe est repéré par l'angle $\alpha_{1}$ et sa fin par $\alpha_{2}$ avec $a_{2}=a_{1}+b$ ( $b$ étant l'amplitude circonférentielle du lobe). ou bien

- les coordonnées cartésiennes $(\mathrm{x}, \mathrm{y})$ du centre de l'arbre par rapport au repère $(O, \vec{x}, \vec{y})$.

L'épaisseur du film fluide s'écrit alors : $\mathrm{h}=\mathrm{C}\left(1+\varepsilon \cos \theta^{*}\right)$ ou bien $\mathrm{h}=\mathrm{C}+(\mathrm{x} \cos \theta+\mathrm{y} \sin \theta)$

Les hypothèses générales de la mécanique des films minces permettent de simplifier les équations de la mécanique des milieux continus et d'obtenir, dans le cas d'un palier en écoulement laminaire, une équation aux dérivées partielles du second ordre en terme de pression :

Equation de Reynolds :

$\frac{1}{R^{2}} \frac{\partial}{\partial \theta}\left[\frac{h^{3}}{\mu k_{x}} \frac{\partial P}{\partial \theta}\right]+\frac{\partial}{\partial z}\left[\frac{h^{3}}{\mu k_{z}} \frac{\partial P}{\partial z}\right]=\frac{\omega}{2} \frac{\partial h}{\partial \theta}+\dot{x} \cos \theta+\dot{y} \sin \theta$

Les divers paramètres (cf figure 1) intervenant dans l'équation sont les suivants :

- $\mathrm{R}$ : rayon du palier.

$-\omega$ : vitesse de rotation.

$-\mu$ : viscosité de l'huile.

$-\theta$ : coordonnée angulaire dont l'origine est située sur la ligne des centres.

$-\mathrm{z}$ : coordonnée longitudinale.

— $\mathrm{h}$ : épaisseur du film d'huile $=\mathrm{C}(1+\varepsilon \cos \theta)$.

$\ldots(\dot{x}, \dot{y}):$ vitesse du centre de l'arbre par rapport au coussinet.

$-\varepsilon$ : excentricité relative.

Dans le cas de fluides peu visqueux, de jeux de fonctionnement importants, de vitesse de rotation et gradients de pression élevés, l'écoulement n'est plus laminaire et les effets d'inertie ne sont plus négligeables, ces effets doivent être introduits dans les calculs. Ces conditions de fonctionnement apparaissent notamment dans les calculs de joints d'étanchéité annulaires (sur les turbopompes). Nous allons présenter dans les paragraphes suivants une synthèse des travaux réalisés sur ce thème au LMS $([3,4]$ et $[5,6])$.

\subsection{Prise en compte des turbulences}

Pour des écoulements à grands nombres de Reynolds, il apparaît une formation de tout un ensemble de mouvements imbriqués les uns dans les autres. Les échelles de longueur, de vitesse et de temps sont telles que les fluides peuvent être assimilés à un milieu continu. Nous pouvons alors obtenir les équations de Navier-Stokes par intégration de l'expres- sion du tenseur de contrainte défini pour un fluide newtonien dans l'expression des équations de la dynamique. Cependant la résolution directe des équations de Navier-Stokes conduirait à un découpage beaucoup trop fin vis-à-vis de la capacité des moyens de calculs actuels.

Reynolds propose dès 1878 de décomposer l'écoulement en une partie moyenne et une partie fluctuante. Il obtient, dans le cadre d'un fluide incompressible, le système des équations de Reynolds dans lequel les effets de la turbulence apparaissent dans un terme appelé tensions de Reynolds. Boussinesq [7] a proposé en 1887 une formulation permettant de reporter la turbulence dans un terme de viscosité turbulente $\bar{\mu}_{i}$.

Plusieurs approches sont utilisées pour caractériser cette viscosité turbulente : les approches à zéro équation et les modèles à une ou deux équations de transport supplémentaire.

Les trois principaux modèles à zéro équation sont :

- les hypothèses de longueur de mélange,

- les lois de paroi qui permettent de décrire le profil des vitesses en fonction de la distance à la paroi,

- la théorie du Bulk Flow qui est une analyse globale de l'écoulement turbulent, analyse basée sur les lois de frottement de Blasius.

Le modèle à deux équations le plus utilisé est le modèle $(K \varepsilon), K$ étant l'énergie cinétique de la turbulence et $\varepsilon$ le taux de dissipation.

Le modèle de turbulence qui a été utilisé par F. Simon [8] et repris par V. Lucas s'appuie sur la relation de Reichard [9] (au voisinage des parois) et sur celle de Clauser [10] (éloigné des parois).

Ce modèle de viscosité turbulente est introduit dans les équations de la mécanique des fluides appliquées dans le cadre de l'hypothèse du film mince. Ces équations sont intégrées suivant l'épaisseur du film, ceci pour obtenir, dans le cas d'un film annulaire, une équation en variable de pression analogue à l'équation de Reynolds :

$\frac{1}{R^{2}} \frac{\partial}{\partial \theta}\left[\frac{h^{3}}{\mu k_{x}} \frac{\partial P}{\partial \theta}\right]+\frac{\partial}{\partial z}\left[\frac{h^{3}}{\mu k_{z}} \frac{\partial P}{\partial z}\right]=\frac{\omega}{2} \frac{\partial h}{\partial \theta}+\dot{x} \cos \theta+\dot{y} \sin \theta$

avec $\mathrm{k}_{\mathrm{x}}$ et $\mathrm{k}_{\mathrm{z}}$ deux coefficients de viscosité turbulente.

Ces coefficients dépendent des paramètres de l'écoulement suivants : nombre de Reynolds local Re et gradients de pression en $\mathrm{z}$ et en $\theta$. Ils sont calculés pour toute une plage de valeurs de ces trois paramètres de l'écoulement. Une tabulation de $k_{\mathrm{x}}$ et $\mathrm{k}_{\mathrm{z}}$ peut être ensuite utilisée pour effectuer un calcul de champ de pression.

Une formulation linéarisée développée par Constantinescu [11] est aussi utilisée dans le cas d'écoulement hydrodynamique (écoulement de Couette prépondérant vis-à-vis de l'écoulement de Poiseuille) pour laquelle :

$\mathrm{k}_{\mathrm{x}}=12+0,00136 \mathrm{R}_{\mathrm{e}}^{0,9}$

$k_{z}=12+0,0043 R_{e} 0,96$

Ces modèles sont utilisés pour calculer les caractéristiques des joints annulaires.

\subsection{Effets d'inertie du fluide}

Il est possible de montrer que le rapport entre les forces d'inertie et les forces de viscosité dans un palier (ou joint) est représenté par le nombre de Reynolds modifié : $\mathrm{R}_{\mathrm{e}}{ }^{*}=\mathrm{R}_{\mathrm{e}} \mathrm{C} / \mathrm{R}$ (C étant le jeu radial et $\mathrm{R}$ le rayon du mécanisme). 
Lorsque le nombre de Reynolds modifié est grand, les forces d'inertie deviennent prépondérantes vis-à-vis des effets visqueux. Dans le cas du calcul d'un joint annulaire avec effets d'inertie, l'écoulement est décomposé en un système d'équations non linéaires d'ordre zéro (caractérisant le point d'équilibre statique) et un système d'équations linéaires d'ordre un (caractérisant le comportement dynamique). Les termes d'inertie sont modélisés en fonction de la vitesse moyenne du fluide par une méthode développée par Constantinescu [12].

\subsection{Détermination du champ de pression}

L'intégration de l'équation de Reynolds par une méthode numérique (différences finies) ou moyennant quelques hypothèses, analytique (palier court ou palier long) permet d'obtenir le champ de pression. Les charges exercées par le fluide sur l'arbre sont alors obtenues par intégration de ce champ de pression.

\section{III — COMPORTEMENT DYNAMIQUE}

\subsection{Introduction}

L'influence d'un élément fluide dans l'étude d'une ligne d'arbre de machines tournantes est caractérisée par les forces hydrodynamiques engendrées par le champ de pression du film fluide. Ces forces hydrodynamiques sont des fonctions non linéaires de la position et de la vitesse du centre de l'arbre dans l'élément fluide. En général le rotor est soumis à la superposition d'une charge statique (poids, force d'engrènement, tension d'une courroie...) et d'une charge dynamique (balourd, vibration d'arbre...). La charge statique impose certaines conditions de fonctionnement. Elle est caractérisée notamment par une position d'équilibre statique (équilibre stable ou parfois instable). La charge dynamique crée, lorsqu'elle est périodique, un mouvement orbital (orbite non circulaire) de l'arbre "autour" de la position d'équilibre statique. Nous allons développer les deux types d'approches qui peuvent être envisagées afin de modéliser ce comportement : une approche linéaire et une non linéaire.

\section{- 3.2 Théorie linéaire}

La théorie linéaire est basée sur une hypothèse de linéarisation au voisinage d'une position d'équilibre. Cela n'est en réalité possible que pour des éléments ayant une position d'équilibre statique. Ainsi les films amortisseurs non montés sur ressorts ne possèdent pas de position d'équilibre, il est donc impossible de leur appliquer ce modèle.

Pour une position d'équilibre statique donnée, imposée par la charge statique $\mathrm{W}_{0}$, nous nous plaçons dans le cadre des hypothèses des petits déplacements du centre de l'arbre. Les forces du fluide sur l'arbre sont alors linéarisées en fonction des accélérations (si les inerties du fluide ne sont pas négligées), des vitesses et des déplacements du centre de l'arbre. Ainsi, au voisinage d'une position d'équilibre statique $\left(\mathrm{x}_{0}\right.$, $\mathrm{y}_{0}$ ) de l'arbre dans le palier, un développement au premier ordre est effectué et les relations s'écrivent sous la forme matricielle suivante :

$$
\left\{\begin{array}{l}
f_{x} \\
f_{y}
\end{array}\right\}=-\left[a_{i j}\right]\left[\begin{array}{l}
\Delta x \\
\Delta y
\end{array}\right\}-\left[b_{i j}\right]\left[\begin{array}{l}
\Delta \dot{x} \\
\Delta \dot{y}
\end{array}\right\}-\left[m_{i j}\right]\left[\begin{array}{l}
\Delta \ddot{x} \\
\Delta \ddot{y}
\end{array}\right\}
$$

où $\mathrm{f}_{\mathrm{x}}$ et $\mathrm{f}_{\mathrm{y}}$ sont les forces additionnelles engendrées par les déplacements, les vitesses et les accélérations de déplacements élémentaires.
Les coefficients dynamiques $a_{i j}, b_{i j}$, et $m_{i j}$ correspondent respectivement aux raideurs, aux amortissements et aux masses ajoutées du film lubrifiant au voisinage du point d'équilibre statique.

La linéarisation du comportement du palier autour d'une position d'équilibre offre de nombreux avantages. Elle permet en premier lieu d'exprimer les coefficients de raideur et d'amortissement du palier. Ces coefficients dynamiques sont souvent utilisés dans les calculs des structures de façon à introduire l'effet du palier le plus simplement possible et de calculer ainsi les modes de fonctionnement ainsi que la réponse des rotors à des sollicitations dynamiques.

La théorie linéaire permet d'établir des cartes de stabilité qui définissent, avec un modèle d'arbre simplifié, la stabilité du point de fonctionnement. Il faut cependant connaître les limites de cette modélisation :

— un palier soumis à des sollicitations dynamiques importantes voit son comportement sortir du domaine linéaire. L'hypothèse des petits déplacements n'est plus vérifiée, les coefficients de raideur et d'amortissement ne sont plus représentatifs de l'action du fluide sur l'arbre. Il faut alors effectuer une étude non linéaire.

- Ces coefficients ne sont définis que pour des conditions de fonctionnement particulières : pour une vitesse, une charge, des géométries,... fixées.

- le comportement des paliers qui fonctionnent dans la zone instable ne peut être prévu. Il n'est pas possible d'associer des coefficients dynamiques à un palier qui ne possède pas de position d'équilibre.

\section{- 3.3 Analyse non linéaire}

L'hypothèse des petits déplacements ne permet pas de prévoir le comportement d'une ligne d'arbre au-delà du seuil de stabilité ou sous l'effet de fortes charges dynamiques. Il est alors nécessaire de résoudre simultanément l'équation de Reynolds et les équations de mouvement. Il s'agit d'un calcul itératif dans le temps nécessitant la résolution de l'équation de Reynolds à chaque instant et ceci pour chaque élément fluide. Ce calcul est très coûteux en temps de calcul et il est essentiellement utilisé dans les cas où les effets de la turbulence sont faibles et lorsque les inerties du fluide sont négligeables (dans le cas des joints, en particulier, il n'est pas actuellement envisageable de faire du calcul dynamique non linéaire).

\section{- 3.4 Exemple de calcul non linéaire}

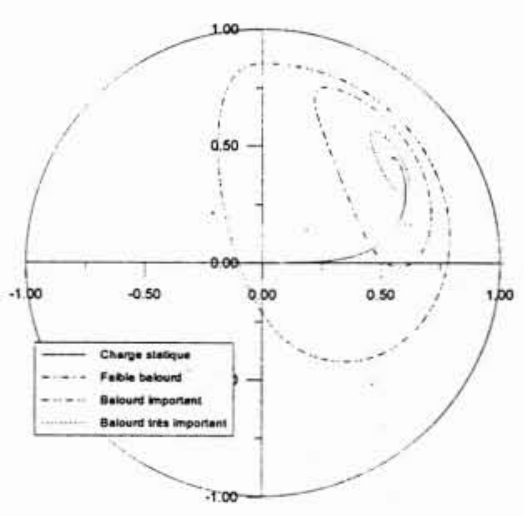

2. Trajectoires non linéaires du centre de l'arbre pour 4 valeurs de balourd. 
Nous avons représenté sur la figure 2 les trajectoires non linéaires du centre de l'arbre chargé statiquement et soumis à différentes valeurs de balourd. Un calcul linéaire conduirait à l'obtention d'ellipses centrées sur la position d'équilibre. Le calcul obtenu par la méthode linéaire dans le cas du chargement à faible balourd serait proche, mais il conduirait à des résultats complètement erronés pour les cas de forts balourds car dans ce cas la trajectoire linéaire sort du domaine admissible défini par le jeu radial.

La modélisation non linéaire permet de tenir compte du comportement réel de l'élément fluide. La modélisation d'arbre utilisée classiquement en lubrification reste très simplifiée (corps rigide). Cette approche présente l'intérêt de séparer les différents problèmes et de se focaliser sur la réponse du seul élément fluide. Cependant il est maintenant nécessaire dans les turbomachines d'avoir un fonctionnement nominal à des fréquences supercritiques. L'étude du comportement de l'ensemble lors d'un passage par une fréquence de résonance ne peut alors s'envisager qu'avec un rotor déformable. Sous de telles contraintes les déplacements du rotor deviennent très importants, ce qui conduit souvent les supports fluides à avoir un comportement fortement non linéaire. On a donc développé des méthodes de calcul permettant de coupler une analyse dynamique non linéaire des paliers avec une modélisation d'un arbre souple caractérisé par sa base modale. Cette approche a été développée en collaboration avec le Laboratoire de Mécanique des Structures de l'INSA de Lyon ([13] et [14]). Nous allons présenter quelques résultats obtenus avec ce modèle.

\section{IV — ROTOR FLEXIBLE MONTÉ SUR FILM AMORTISSEUR}

Les caractéristiques principales de l'arbre de cette étude sont :

- une masse assez faible,

- une charge statique faible,

- une charge dynamique de type balourd avec une vitesse

de rotation assez élevée,

— un arbre souple conduisant à des phénomènes dyna-

miques importants (vitesses critiques).

Kassai [13] a montré expérimentalement et numériquement que pour un rotor de ce type monté dans deux roulements rigides, le passage de la vitesse critique est très délicat, les amplitudes devenant trop importantes. Afin de limiter cellesci, un montage dans un film amortisseur (Squeeze Film Damper) a été envisagé. Nous allons présenter dans un premier temps certaines caractéristiques du film amortisseur, puis un calcul non linéaire de l'ensemble sera effectué en tenant compte des flexibilités de l'arbre. Les résultats obtenus seront comparés avec des résultats expérimentaux.

\subsection{Le film amortisseur}

L'idée de base de ce type de palier est de monter un roulement à billes dans un palier fluide. La rotation de l'arbre est supportée par le roulement alors que le palier fluide joue le rôle d'un amortisseur visqueux. Le mouvement de la bague dans le palier se réduit à une translation, il n'y a pas de rotation entre la bague extérieure du roulement et son logement.

Deux technologies de paliers avec film amortisseur sont possibles. Un premier montage consiste à monter en parallèle avec le film amortisseur un ressort qui permet de donner une certaine raideur au palier ([15] et [16]). Il ne sera pas étudié ici. La deuxième configuration (Figure 3) consiste à laisser le système libre, certains problèmes de raideur du palier se posent alors. En effet, la création d'un champ de pression pouvant assurer la portance nécessite un seuil de perturbations minimales.

\subsection{Résultats}

Nous allons présenter les résultats relatifs à un arbre flexible monté sur deux types de paliers. A une extrémité, nous avons un roulement à billes modélisé par une raideur importante et à l'autre extrémité un roulement à billes dans un film amortisseur (figure 4).

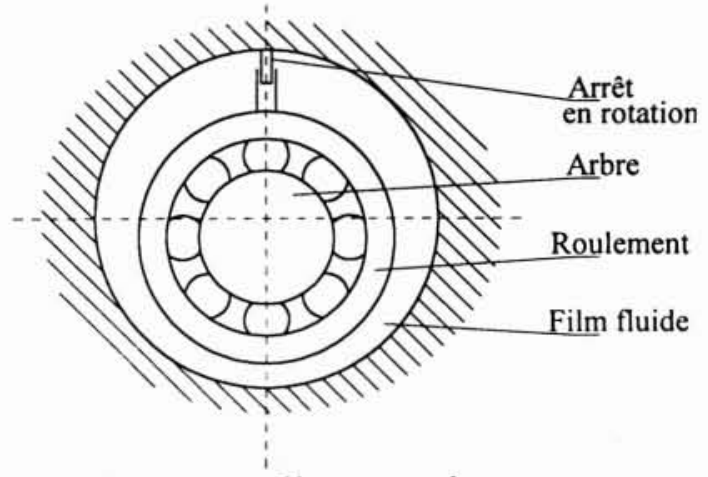

3. Film amortisseur.

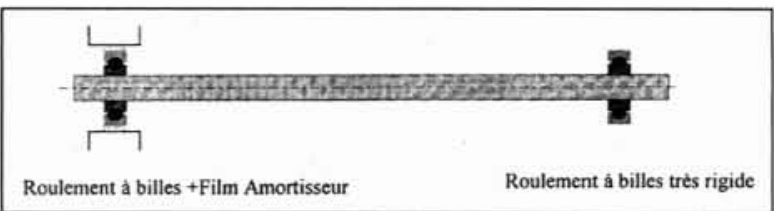

4. Rotor de l'étude.

Le rotor a une longueur de $0,94 \mathrm{~m}$ pour un diamètre de $0,06 \mathrm{~m}$.

Le film amortisseur étudié a les caractéristiques géométriques suivantes :

Longueur $l=0,015 \mathrm{~m}$

Rayon $\mathrm{R}=0,0448 \mathrm{~m}$

Jeu $C=0,0510^{-3} \mathrm{~m}$.

Le comportement du rotor est obtenu par simulation d'une montée en vitesse, les déplacements au milieu du rotor et dans le film amortisseur seront présentés en fonction de la vitesse de rotation.

Les courbes de résultats représentent les normes des vecteurs positions de l'arbre par rapport au bâti. Ces vecteurs ont pour origine le point $\mathrm{O}$ (centre géométrique du palier). Le centre de l'arbre décrit une orbite fermée non centrée en $\mathrm{O}$, ce qui explique qu'en fonction de la vitesse les courbes présentent des oscillations.

\subsection{Influence du jeu radial}

Deux valeurs du jeu dans le film amortisseur ont été étudiées : $0,05 \mathrm{~mm}$ et $0,1 \mathrm{~mm}$. Les résultats relatifs à une montée en vitesse de $8000 \mathrm{tr} / \mathrm{min}$ à $14000 \mathrm{tr} / \mathrm{min}$ en $20 \mathrm{~s}$ sont présentés sur les figures 5 et 6 . L'augmentation du jeu s'accompagne d'une baisse très sensible des amplitudes au milieu du rotor, les amplitudes à la résonance étant très atténuées. Cela se fait au détriment des déplacements dans le palier qui deviennent beaucoup plus importants. La fréquence de résonance est diminuée lorsque le jeu augmente. Les forces exercées par le fluide sur le bâti ont aussi été étudiées [14], elles sont fortement diminuées par un accroisse- 
ment du jeu dans le film amortisseur. L'étude présentée ici permet de mieux comprendre certains phénomènes. Une étude simplifiée avec rotor rigide aurait conduit à des résultats complètement opposés. En effet l'augmentation du jeu se serait traduite par une augmentation des amplitudes de l'arbre et des forces transmises.

Au contraire, les calculs effectués avec un rotor flexible mettent en évidence l'accroissement de l'efficacité du film amortisseur avec l'évolution du jeu. Cela s'explique assez simplement : une augmentation du jeu s'accompagne de déplacements plus importants au niveau du film donc une dissipation d'énergie accrue et un meilleur comportement de l'ensemble à la résonance. Ces résultats sont intéressants car l'arbre est suffisamment flexible pour que les déplacements dans le palier soient négligeables par rapport aux déplacements au milieu de l'arbre. Un film amortisseur qui serait monté sur un arbre très rigide aurait une influence contraire sur cet arbre (les mouvements du milieu de l'arbre étant du même ordre de grandeur que ceux dans le palier). Des conclusions complètement identiques peuvent être obtenues lorsque l'on diminue la viscosité du fluide d'alimentation [14].

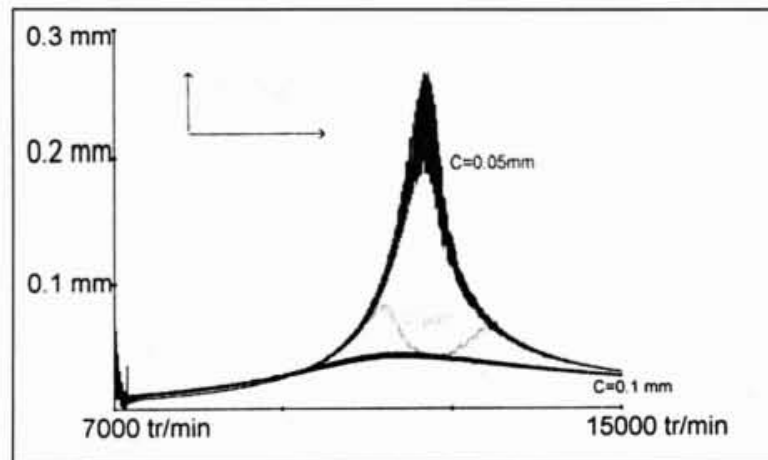

5. Amplitude au centre du rotor en fonction de la vitesse de rotation.

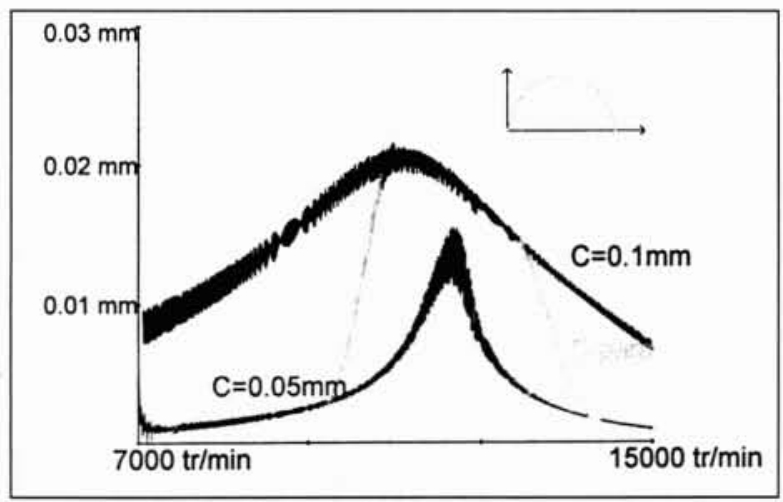

6. Amplitude dans le film amortisseur en fonction de la vitesse de rotation.

\subsection{Film amortisseur actif}

Les conclusions du paragraphe précédent nous ont conduit à envisager la conception d'un film amortisseur adaptatif [17]. En effet, loin des vitesses critiques, il est préférable de diminuer les vibrations dans le film amortisseur (jeu faible ou viscosité importante). Par contre, au voisinage d'une vitesse critique, le film amortisseur doit avoir un jeu plus élevé et une viscosité plus faible.
Le film amortisseur est calculé par une méthode palier court, sans ressort de centrage. Le jeu variable du film amortisseur est obtenu par une bague conique réglable suivant un paramètre $\mathrm{x}$ qui évolue en fonction de la vitesse de rotation (Figure 7). Une évolution parabolique du jeu en fonction de la vitesse de rotation est effectuée.

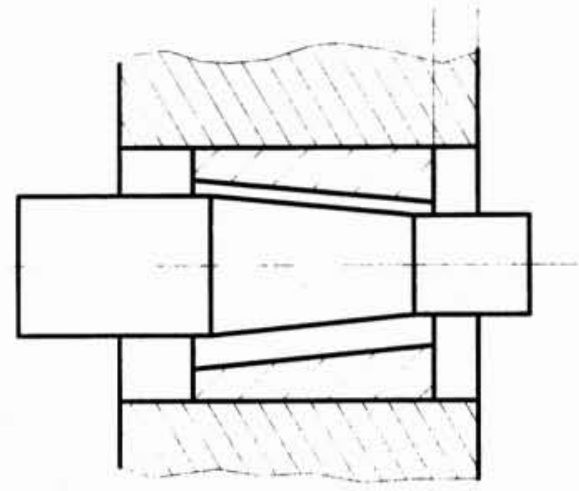

\section{Film amortisseur actif}

Les courbes $\mathrm{C}_{\text {para }}$ des figures 5 et 6 présentent les réponses lorsque le jeu varie (courbes $\mathrm{C}$ para). Nous remarquons que la variation du jeu permet de passer de la réponse faible jeu à la réponse jeu important tout en optimisant les amplitudes.

D'autres types de technologies dédiées au contrôle actif des lignes d'arbres ont été étudiées. Nous avons notamment développé une approche numérique permettant de modéliser un film amortisseur alimenté par fluide électrorhéologique [18]. Ces lubrifiants ont une viscosité qui évolue en fonction du champ électrique dans lequel ils évoluent. Les premiers résultats sont prometteurs mais la technologie électrorhéologique en est encore à ces débuts. Les fluides électrorhéologiques commercialisés aujourd'hui ne sont pas assez performants et nécessitent d'être améliorés. Ils sont le siège de phénomènes physico-chimiques complexes et réclament, pour être améliorés, des connaissances approfondies en chimie des polymères, en chimie organique synthétique et chimie des surfaces. La mise au point de fluides électrorhéologiques performants pose donc de nombreux problèmes. La conception de mécanismes électrorhéologiques demande une approche spécifique, il faut repenser les mécanismes actuels en tenant compte des propriétés de ces nouveaux fluides :

- pouvoir appliquer des différences de potentiels importantes,

- éviter les zones mortes pouvant conduire à une sédimentation des particules.

Il faut par ailleurs optimiser les algorithmes de contrôles actifs et mettre au point des alimentations électriques adaptées à ces nouveaux composants (tension, fréquence, commande...).

\section{$\mathrm{V} \square$ CONCLUSION}

Le travail présenté ici est une synthèse des différentes approches pouvant être effectuées afin de caractériser dynamiquement le comportement d'une ligne d'arbre montée sur éléments fluides. Les paliers peuvent avoir un comportement considéré en première approximation comme linéaire, ils sont alors modélisés par des matrices de raideur, d'amortissement et éventuellement de masse ajoutée. Par contre lorsque les hypothèses de petits déplacements ne sont plus 
vérifiées il est nécessaire d'effectuer une analyse non linéaire. On présente en particulier dans ce travail les résultats d'un calcul effectué en couplant le comportement flexible d'une ligne d'arbre (lors d'un passage à la résonnance) avec le comportement non linéaire d'un film amortisseur. Ces différents résultats ont par ailleurs été validés par une campagne d'essais effectuée au LMS de l'INSA de Lyon [13-14].

Les principales conclusions de ces premiers travaux ont mis en évidence l'intérêt que peut présenter le contrôle des caractéristiques d'un support fluide. Nous avons développé quelques technologies de paliers actifs. Nous sommes actuellement en train de modéliser un palier monté dans un support mobile contrôlé à grande fréquence de façon à atténuer les perturbations dynamiques s'exerçant sur les lignes d'arbre. Les premiers résultats sont très encourageants.

\section{Bibliographie}

[1] ARGHIR M.; “" Modélisation du comportement dynamique des joints annulaires rainures à fluide incompressible "Thèse de l'Université de Poitiers, 11 décembre 1996

[2] ARGHIR M., FRENE, J., " Inclusion of roughness effects in perturbated Navier Stokes rotordynamic method ". ASME, Jnal of Tribology, 119 , july 1997

[3] SIMON F., FRENE J.,"Rotordynamic coefficients for turbulent annular misaligned seals". The third International Symposium on transport phenomena and dynamics of rotating machinery (ISROMAC 3) Honolulu Hawaiii, Vol. 2 Dynamics, 1990, pp. 289-304

[4] SIMON F., FRENE J., "Analysis for Incompressible Flow in Annular Pressure Seals". ASME Journal of Tribology, vol. 114, 1991, pp.431438.

[5] LUCAS V., DANAILA S., BONNEAU O., FRENE J., "Roughness Influence on turbulent flow through annular seals". Journal of Tribology ASME, vol 116, April 94, pp 321.

[6] V. LUCAS, O. BONNEAU, J. FRENE "Roughness Influence on the Turbulent Flow Through Annular Seals Including Inertia Effects". Journal of Tribology ASME, vol. 118 January 1996, pp 175-182
[7] BOUSSINESQ J.,"Essai sur la théorie des eaux courantes". Mémoires présentés par divers savants à l'Académie des Sciences de l'Institut de France. Sci. Math. Phys., tome 23, 1887, p 46-47.

[8] SIMON F., "Comportement dynamique des joints annulaires à fuite Modélisation des forces d'inertie convective en régume turblent stationnaire et instationnaire". Thèse de l'Université de Poitiers, soutenue le 30 novembre 1989

[9] REICHARD H.,"Vollständige Darstellung der turbulenten geschwindigkeitsverteilung in glatten leitungen in glatten leitungen". Z. angew. Math. Mech., 1951, Vol. 31, n7, p208-219.

[10] CLAUSER F. H., "The turbulent boundary-layer". Advances in applied mechanics, 1965, vol.4, pp 1-51

[11] CONSTANTINESCU, V.N., PAN, C.H.T., SMALLEY, A.J., VOHR, J.H., "Lubrication phenomena in a film of low kinematic viscosity". Rev. Roum. Sc. Techn. Mech. Appl., Vol 15, N², 1970, pp 479-502.

[12] CONSTANTINESCU V. N., "On the Influence of Inertia Forces in Turbulent and Laminar Self-Acting Films", Transactions of the A.S.M.E., Journal of Lubrication Technology, July 1970, pp.473-481.

[13] KASSAI A., "Contribution à l'Etude Dynamique des Rotors Amortis". Thèse de Doctorat de l'INSA de Lyon soutenue le 6 juillet 1989

[14] BONNEAU O., KASSAI A., FRENE J., DER HAGOPIAN J., "Dynamical behavior of an elastic rotor with squeeze film damper" EUROTRIB. HELSINKI FINLANDE juin 89 Proceeding Vol.4, pp 145-149.

[15] PAN C.H.T., TONNESEN J., "Excentric operation of the squeeze film damper". ASME, Jnal. of Lub. Techn., Vol 100, July 1978, pp 369 377.

[16] HAHN E.J., "Stability and unbalance response of centrally preloaded rotors mounted in journal and squeeze film bearings". ASME, Jnal. of Lub. Techn., Vol 101, April 1979, pp 120-128.

[17] O, BONNEAU, J. FRENE "Numerical study of a flexible rotor mounted in an active squeeze film damper". Fourth International Conference On Rotor Dynamics, IFTOM 1994 Chicago pp 327-331.

[18] B. PECHEUX, O. BONNEAU, J. FRENE, "Investigation about Electro-Rheological Squeeze Film Damper Applied to Active Control of Rotor Dynamic" . International Journal of Rotating Machinery, Vol. 3, issue 1, January 1997. 